\title{
A Systematic Review on the Association of D-dimer Levels with the Disease Severity and Mortality of COVID-19
}

\author{
Gamaliel Issamar S. De Vera ${ }^{\mathrm{a}, \mathrm{i}}$, Christian Miguel D. Aglipay, \\ Gene Abbygaile Y. Aguas c,i , Renz Marion C. Evangelista, ${ }^{\mathrm{d}, \mathrm{i}}$, \\ Alyssa Marie G. Francisco ${ }^{\mathrm{e}, \mathrm{i}}$, Alyssa Mikhail B. Le ${ }^{\mathrm{f}, \mathrm{i}}$, \\ Cathleen Dianne O. Salazar, ${ }^{\mathrm{g}, \mathrm{i}}$, Vinz Joshua B. Sy,i \\ a gsdevera@ust.edu.ph, ${ }^{\mathrm{b}}$ christianmiguel.aglipay.pharma@ust.edu.ph, ${ }^{\mathrm{c}}$ geneabbygaile.aguas.pharma@ust.edu.ph, \\ d renzmarion.evangelista.pharma@ust.edu.ph, ${ }^{\mathrm{e}}$ alyssamarie.francisco.pharma@ust.edu.ph, \\ f alyssamikhail.le.pharma@ust.edu.ph, ${ }^{\mathrm{g}}$ cathleendianne.salazar.pharma@ust.edu.ph, \\ ${ }^{\mathrm{h}}$ vinzjoshua.sy.pharma@ust.edu.ph
}

${ }^{i}$ Department of Medical Technology, Faculty of Pharmacy, University of Santo Tomas, España, Manila Philippines

\begin{abstract}
COVID-19 (coronavirus disease 2019), which is caused by the severe acute respiratory syndrome coronavirus 2 (SARS-CoV-2), is a disease that originated from Wuhan, China that can be spread via droplet and airborne transmission, and direct contact. In its diagnosis, RT-PCR analysis of nasopharyngeal swabs and viral sequencing of respiratory or blood samples are utilized. However, due to the gaps in the pathophysiology of the disease, early detection and monitoring of COVID-19 remain insufficient. Thus, the researchers sought to further understand the importance of D-dimer, a by-product of fibrin breakdown, as a potential biomarker for COVID-19 disease severity and mortality, as well as establish its relationship with pre-existing COVID-19-related illnesses. Moreover, a systematic review was performed in order to collate existing evidence and fulfill the research objectives. The Preferred Reporting Items for Systematic Reviews and Meta-Analysis (PRISMA) was utilized to ensure the quality of the data retrieval and to serve as a guide for gathering and screening relevant research articles. The studies used were those that were translated or were written in English and published between January 1, 2020 and January 31, 2021. Furthermore, six (6) screening stages were conducted to ensure that the studies used for data analysis were unbiased and explicit in terms of inclusion and exclusion criteria. The mined D-dimer concentrations above the normal value $(\geq 0.50$ $\mu \mathrm{g} / \mathrm{mL}$ )showed that an increase in D-dimer levels is associated with increasing COVID-19 severity and mortality. Mild to moderate patients had a median value of $0.505 \mu \mathrm{g} / \mathrm{mL}$, whereas severe and critical cases had median values of $0.80 \mu \mathrm{g} / \mathrm{mL}$ and $3.86 \mu \mathrm{g} / \mathrm{mL}$, respectively. In terms of mortality, D-dimer concentrations were similarly shown to be significantly higher in non-survivors of COVID-19, with a median value of $6.34 \mu \mathrm{g} / \mathrm{mL}$, as compared to $0.94 \mu \mathrm{g} / \mathrm{mL}$ for survivors. It was found that D-dimer is a nonspecific biomarker for the severity and mortality of COVID-19 since some comorbidities could potentially contribute to the rise in D-dimer levels. D-dimer levels were significantly higher in severe to critical cases than in mild to moderate states. Non-survivors also had remarkably higher D-dimers than survivors. Further research is needed to establish the reliability of D-dimer as a COVID-19 diagnostic marker. Patients with elevated D-dimers are encouraged to be constantly monitored to avoid clinical deterioration.
\end{abstract}




\section{Introduction}

As a novel virus, World Health Organization (2020) verified severe acute respiratory syndrome coronavirus 2 (SARS-CoV-2) as the causative agent of coronavirus disease 2019 (COVID-19) infection. Discovered in Wuhan, China during the month of December 2019, more than 200 million COVID-19 cases were confirmed worldwide, with more than 4 million cases contributing to mortality rate caused by COVID-19 (World Health Organization, 2021). With regards to the mode of transmission, Rothan and Byrareddy (2020) mentioned that the virus is transmitted by means of droplets, as $\mathrm{Zu}$ et al. (2020) discussed that several confirmatory laboratory methods for COVID-19 may be performed such as real-time reverse transcriptase polymerase chain reaction (rRT-PCR) and viral gene sequencing. Recently, vaccination has already been administered, reaching more than 6 billion vaccine doses in September 2021 (WHO, 2021). On the other hand, D-dimer, as defined by Bounds and Kok (2020), is both a fragment produced from fibrin degradation and a byproduct of blood coagulation. As a laboratory parameter, it is measured in patients suspected to have clotting disorders (Medline Plus, 2020).

Association between COVID-19 infection and D-dimer levels were established in several journals, and Dugar et al. (2020) added that clinical manifestations that correlate COVID-19 from D-dimer include intense inflammation and acquired thrombus-related complications. It was also stated by Cao et al. (2020) that D-dimer is a reliable biomarker for categorizing COVID-19 severity and presence of clotting within the body system. Subsequently, some researchers claimed that coagulation with COVID-19 is induced by the inflammatory response on SARS-CoV-2, resulting in thrombin inflammation and comorbidities associated with thrombosis (Connors \& Levy, 2020). Due to its emergence, further studies are required to analyze the relationship between COVID-19 severity and mortality rate due to COVID-19 and D-dimer levels on COVID-19 patients.

\subsection{Objectives of the Study}

The primary aim of this study was to systematically evaluate different studies to determine the relationship between D-dimer levels and COVID-19 severity and mortality. Specifically, the study aimed to ascertain if D-dimer levels are elevated in individuals diagnosed with COVID-19, evaluate the elevation of D-dimer levels in COVID-19 patients with mild to moderate, severe, and critical conditions, compare the D-dimer levels between COVID-19 survivors and non-survivors, and determine pre-existing conditions associated with elevated D-dimer in COVID-19 patients.

\subsection{Scope and Limitations}

A systematic review was conducted due to the limitations of the pandemic and the nature of objectives. Several methodologies were absent such as performing experimental procedures and acquiring direct respondents for surveys or interviews, yet the study focused on evaluating the correlation of COVID-19 severity (e.g. mild, moderate, severe, critical) and mortality (e.g. survivors, nonsurvivors) to D-dimer levels. Journals used for the study met the inclusion criteria and were retrieved from selected electronic databases which were discussed under the Methodology section.

\subsection{Significance of the Study}

Due to the sudden rise of COVID-19 and prevalence of pneumonia and thrombotic complications that may be associated with COVID-19 infection, the study aimed to pursue on establishing the reliability on D-dimer levels as a biomarker and its significance in diagnosing and categorizing COVID-19 infection, and predicting the mortality of COVID-19. Significant individuals such as physicians, medical technologists, health sectors and organizations, the academe, and future researchers were inferred to primarily be aided with the systematic review. 


\section{Methodology}

The methodology of the study presents the research design, retrieval strategy, inclusion and exclusion criteria, screening, data extraction, and methodology quality assurance. The research design the type of study done, retrieval strategy elaborates on how the studies were collected, the inclusion and exclusion criteria for standards for the gathered studies, screening discusses the procedure of filtering the gathered studies, data extraction shows what information will be used from the screened studies, and methodology quality assurance ensures the proper procedures were followed. These will be further explained below.

\subsection{Research Design}

The researchers performed a systematic review to synthesize and analyze data from the gathered studies. This provides an exhaustive summary of the scholarly literature related to a particular research which aimed to identify the association of D-dimer levels with the disease severity and mortality of COVID-19 by thoroughly comparing different researches to analyze the collected data which ultimately allowed the researchers to come up with a conclusion. Narrative synthesis was primarily employed to explain and summarize the findings, and data were presented through tabulations and verbal interpretation.

\subsection{Retrieval Strategy}

For this systematic review, the literature retrieval was based on the guidelines provided by PRISMA or the Preferred Reporting Items for Systematic Review and Meta-Analyses. The researchers collected research articles from different electronic databases such as Google Scholar, ScienceDirect, and NCBI (PubMed) using the following keywords: coronavirus, COVID-19, and D-dimer. Combinations of terms, including "Correlation of coronavirus/COVID-19 and D-dimer levels", "Association of coronavirus/COVID-19 with D-dimer", and "coronavirus/COVID-19 and D-dimer", were utilized to enhance the specificity of the study. Only research articles that were written or translated in English were included.

\subsection{Inclusion Criteria}

Studies were included if all of the following criteria were met: (1) participants were COVID-19 patients, either symptomatic or asymptomatic; (2) the study was written or translated in English; (3) publishing date was from January 1, 2020 to January 31,2021 ; (4) the study was quantitative in nature and must have specified actual D-dimer values of patients with mild to moderate, severe, and/or critical conditions, as well as those who survived and died; (5) for studies that addressed COVID-19 severity, at least two of the aforementioned severity classifications must have been included for D-dimer comparison to proceed; (6) for studies that tackled COVID-19 mortality, D-dimer for survivors and non-survivors must have been compared; (7) sex and age of patients were specified, as well as the statistical analysis used; and (8) title and abstract were clear, precise, and complete.

\subsection{Exclusion Criteria}

Studies were excluded if any of the following criteria were present: (1) the study was published as a review, case report, or commentary; (2); only the abstract was available; (3) the scope was wide and not focused on the main topic; (4) actual D-dimer values were not specified; and (5) results were not clearly defined.

\subsection{Screening}

A total of 13,900 studies were initially gathered by the researchers. Screening for research articles started with filtering the date of publication from January 2020 to January 2021, removing 4,800 articles. Followed by eliminating 8,300 
studies with ambiguous titles and wider scopes, then 350 articles were excluded due to the absence of abstract. Next, 226 studies were removed from the selection after consideration of the sex and age patients together with the statistical treatment used in the study. The remaining 124 studies were screened based on insufficient data on severity classification and survival comparison, of which 53 studies were withdrawn. Lastly, 51 studies without actual D-dimer values were eliminated, resulting in a total of 20 studies left to be reviewed in Chapter 4.

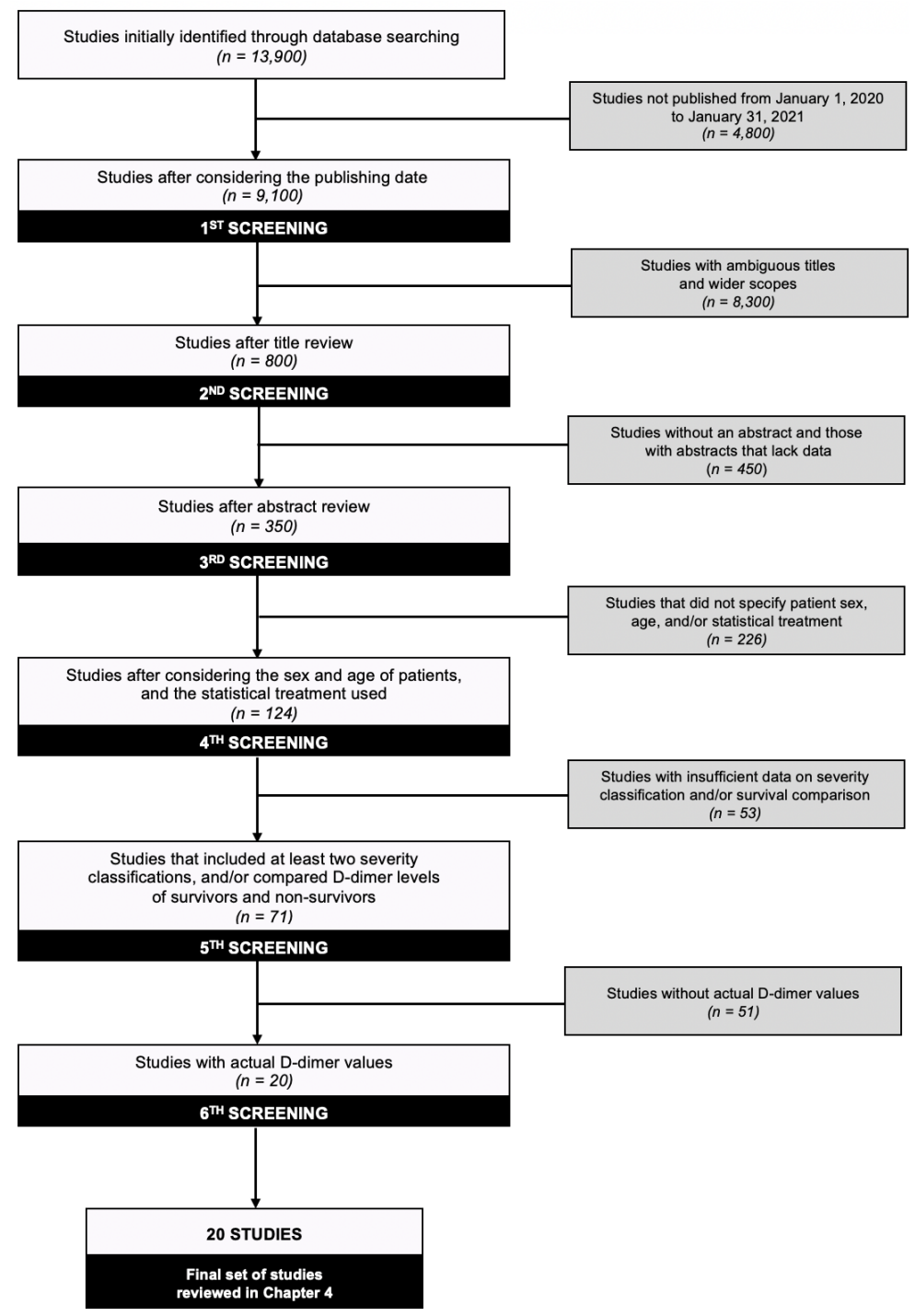

Fig. 1. Screening Procedure Flowchart

\subsection{Data Extraction}

The contents that were extracted from the journals included the research type and design, study period, objectives, research site, exclusion and inclusion standard, sample size, methodology, control group, findings, and conclusion.

\subsection{Methodology Quality Assurance}

The PRISMA framework was used for assurance of formulating the correct flow of systematic review. With the use of the PRISMA checklist, the researchers were assigned to analyze whether there were inconsistencies in the approach of 
the research and for further improvements to be made in the data gathering process. The PRISMA checklist was utilized to validate the quality of methodology used in the study.

\section{Results and Discussion}

\subsection{Background of Research Articles}

Selected studies were filtered according to the inclusion and exclusion criteria discussed in Chapter 2.3 and Chapter 2.4, respectively. The profiles of each of the 20 studies are tabulated in Table 1, showing their title, author, date of publication, study region, and study type.

Table 1. Characteristics of Selected Studies

\begin{tabular}{|c|c|c|c|c|}
\hline Research title & Author & $\begin{array}{l}\text { Date of } \\
\text { publication }\end{array}$ & Study region & Study type \\
\hline $\begin{array}{l}\text { Prevalence and outcomes of D-dimer } \\
\text { elevation in hospitalized patients with } \\
\text { COVID-19 }\end{array}$ & Berger et al. & August 25, 2020 & New York, USA & $\begin{array}{l}\text { Retrospective } \\
\text { Observational }\end{array}$ \\
\hline $\begin{array}{l}\text { Diagnostic performance of initial blood urea } \\
\text { nitrogen combined with D-dimer levels for } \\
\text { predicting in-hospital mortality in COVID-19 } \\
\text { patients }\end{array}$ & Cheng et al. & July 23, 2020 & Wuhan, China & Retrospective \\
\hline $\begin{array}{l}\text { Clinical characteristics of } 140 \text { patients } \\
\text { infected with SARS-CoV-2 in Wuhan, China }\end{array}$ & Dong et al. & February 19, 2020 & Wuhan, China & Retrospective \\
\hline $\begin{array}{l}\text { Diagnostic utility of clinical laboratory data } \\
\text { determinations for patients with severe } \\
\text { COVID-19 }\end{array}$ & Gao et al. & March 17, 2020 & Fuyang, China & Retrospective \\
\hline $\begin{array}{l}\text { The poor prognosis and influencing factors of } \\
\text { high D-dimer levels for COVID-19 patients }\end{array}$ & He et al. & January 19, 2021 & Wuhan, China & $\begin{array}{l}\text { Multicenter } \\
\text { Retrospective }\end{array}$ \\
\hline $\begin{array}{l}\text { A cohort study of } 676 \text { patients indicates } \\
\text { D-dimer is a critical risk factor for the } \\
\text { mortality of COVID-19 }\end{array}$ & Huang et al. & November 9, 2020 & Wuhan, China & Retrospective \\
\hline $\begin{array}{l}\text { Biomarkers and short-term prognosis in } \\
\text { COVID-19 }\end{array}$ & Peiró et al. & January 18, 2021 & Tarragona, Spain & $\begin{array}{l}\text { Retrospective } \\
\text { Observational }\end{array}$ \\
\hline $\begin{array}{l}\text { The value of clinical parameters in predicting } \\
\text { the severity of COVID- } 19\end{array}$ & Shang et al. & May 21,2020 & Wuhan, China & $\begin{array}{l}\text { Single-center } \\
\text { Retrospective }\end{array}$ \\
\hline $\begin{array}{l}\text { D-dimer level is a useful predictor for } \\
\text { mortality in patients with COVID-19: } \\
\text { Analysis of } 483 \text { cases }\end{array}$ & Soni et al. & $\begin{array}{l}\text { November } 17, \\
2020\end{array}$ & Chennai, India & $\begin{array}{l}\text { Single-center } \\
\text { Retrospective }\end{array}$ \\
\hline $\begin{array}{l}\text { Role of hematological parameters in the } \\
\text { stratification of COVID-19 disease severity }\end{array}$ & Taj et al. & January 8, 2021 & Lahore, Pakistan & Retrospective \\
\hline
\end{tabular}


Elevated D-dimer is associated with multiple clinical outcomes in hospitalized COVID-19 patients: A retrospective cohort study

Clinical features and treatment of COVID-19 patients in Northeast Chongqing

D-dimer as a biomarker for disease severity and mortality in COVID-19 patients: A case control study

Dynamic changes of D-dimer and neutrophil-lymphocyte count ratio as prognostic biomarkers in COVID-19

D-dimer level is associated with the severity of COVID-19

D-dimer levels on admission to predict in-hospital mortality in patients with COVID-19

Clinical course and risk factors for mortality of adult inpatients with COVID-19 in Wuhan, China: A retrospective cohort study

\begin{tabular}{|c|c|c|c|}
\hline Wagner et al. & November 8, 2020 & Texas, USA & $\begin{array}{l}\text { Retrospective } \\
\text { Cohort }\end{array}$ \\
\hline Wan et al. & April 1, 2020 & $\begin{array}{l}\text { Northeast } \\
\text { Chongqing, China }\end{array}$ & Retrospective \\
\hline Yao et al. & July 10, 2020 & Wuhan, China & Retrospective \\
\hline Ye et al. & July 3, 2020 & Wuhan, China & $\begin{array}{l}\text { Single-center } \\
\text { Retrospective }\end{array}$ \\
\hline Yu et al. & July 27, 2020 & Wuhan, China & $\begin{array}{l}\text { Retrospective } \\
\text { Cohort }\end{array}$ \\
\hline Zhang et al. & April 19, 2020 & Wuhan, China & $\begin{array}{l}\text { Single-center } \\
\text { Retrospective }\end{array}$ \\
\hline Zhou et al. & March 11, 2020 & Wuhan, China & $\begin{array}{l}\text { Multicenter } \\
\text { Retrospective } \\
\text { Cohort }\end{array}$ \\
\hline
\end{tabular}

All 20 included studies were published in English. Table.1 summarizes the characteristics of each study. 15 studies were performed in China (Huang et al., 2020; Gao et al., 2020; Liu et al., 2020; Shang et al., 2020; Zhou et al., 2020; He et al., 2021; Zhang et al., 2020; Yu et al. 2020; Long et al., 2020; Yao et al., 2020; Fu et al., 2020; Ye et al., 2020; Dong et al., 2020; Cheng et al., 2020; and Wan et al., 2020), whereas other studies were performed in Spain (Peiró et al., 2021), USA (Berger et al., 2020 and Wagner et al., 2020), Pakistan (Taj et al., 2021), and India (Soni et al., 2020). All research articles were published from February 19, 2020 to January 19, 2021, and the majority of them were retrospective studies.

Table 2. Demographic Profile of the Patients in each Study

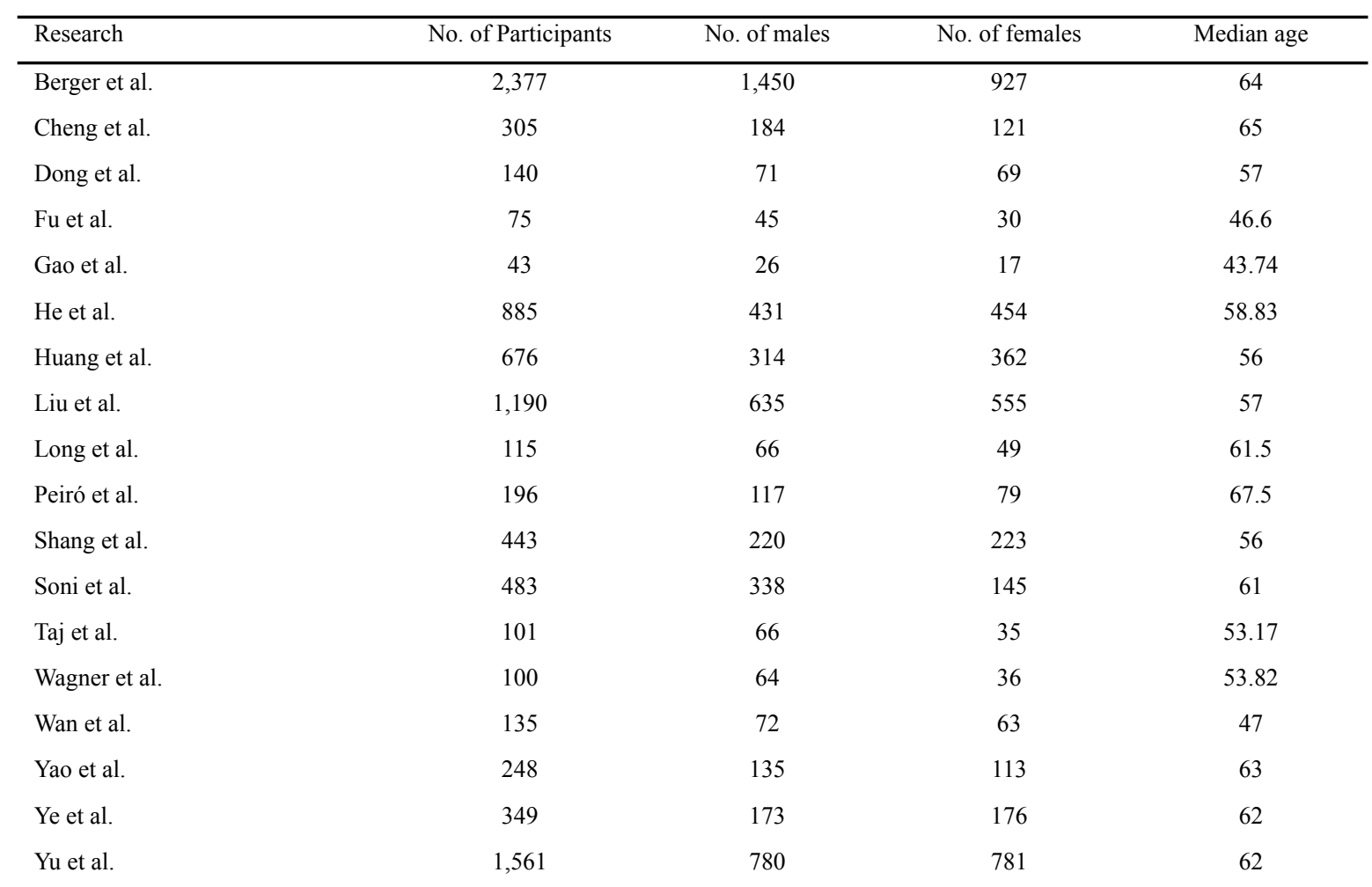




\begin{tabular}{llccc} 
Zhang et al. & 343 & 171 & 172 & 62 \\
Zhou et al. & 191 & 119 & 72 & 56 \\
\hline
\end{tabular}

Table 2 summarizes the demographic profile of all participants in each study. The total sample size from the 20 publications is 9,956 , consisting of 5,477 males and 4,479 females. The overall median age of the participants is 57.92, ranging from 43.74 to 67.5 .

\subsection{Results of the Examined Studies}

Twenty studies were reviewed in this study to provide evidence on the following: relationship of D-dimer levels and COVID-19 severity and mortality, comorbidities associated with elevated D-dimer, possible reasons for increased D-dimer values in COVID-19 patients, and other biomarkers that could aid in the prognosis of COVID-19.

\subsubsection{Relationship of D-dimer levels and the severity of COVID-19}

Among the 20 studies reviewed in this research, 12 studies examined the relationship between D-dimer levels and the severity of COVID-19. These studies recorded the actual D-dimer levels of COVID-19 patients with mild to moderate, severe, and critical conditions. The relative findings are reported in Table 3 and Table 4.

Table 3. Distribution of Patients based on Disease Severity

\begin{tabular}{|c|c|c|c|c|c|}
\hline \multirow[t]{2}{*}{ Research } & \multicolumn{4}{|c|}{ No. of Patients } & \multirow[t]{2}{*}{ Total } \\
\hline & Mild & Moderate & Severe & Critical & \\
\hline Berger et al. & 554 & - & 1,823 & - & 2,377 \\
\hline Dong et al. & \multicolumn{2}{|c|}{82} & 58 & - & 140 \\
\hline Fu et al. & \multicolumn{2}{|c|}{59} & 16 & - & 75 \\
\hline Gao et al. & 28 & - & 15 & - & 43 \\
\hline Liu et al. & \multicolumn{2}{|c|}{841} & 349 & - & 1,190 \\
\hline Long et al. & 39 & - & 48 & 28 & 115 \\
\hline Shang et al. & 304 & - & 139 & - & 443 \\
\hline Soni et al. & 188 & 66 & 133 & - & 387 \\
\hline Taj et al. & 21 & 52 & 20 & 8 & 101 \\
\hline Wan et al. & 95 & - & 40 & - & 135 \\
\hline Yao et al. & \multicolumn{2}{|c|}{90} & - & 50 & 248 \\
\hline Yu et al. & 1,196 & - & 365 & - & 1,561 \\
\hline Total & \multicolumn{2}{|c|}{3,615} & 3,114 & 86 & 6,815 \\
\hline
\end{tabular}

Table 4. The Mined D-dimer Values According to Disease Severity

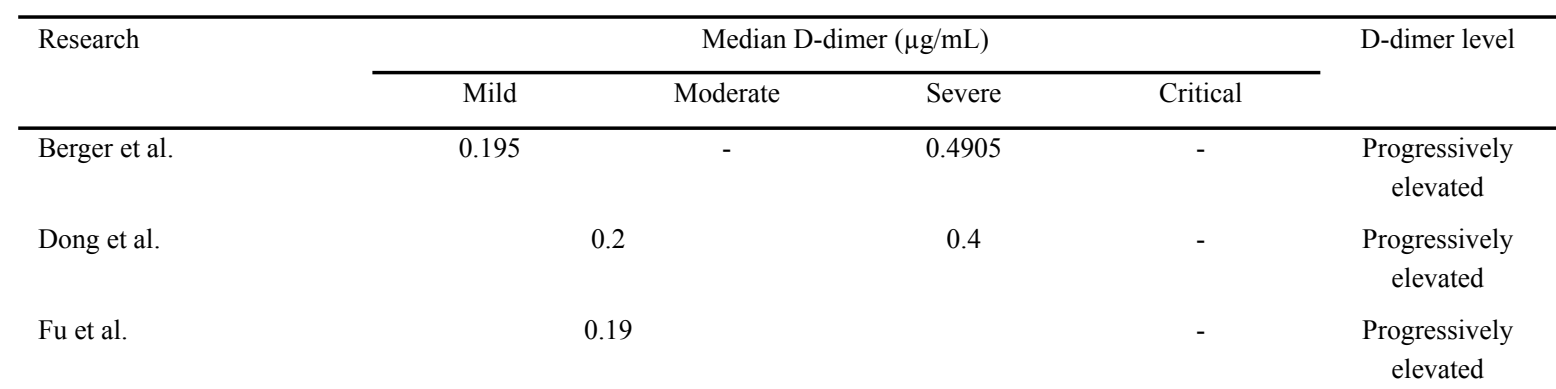




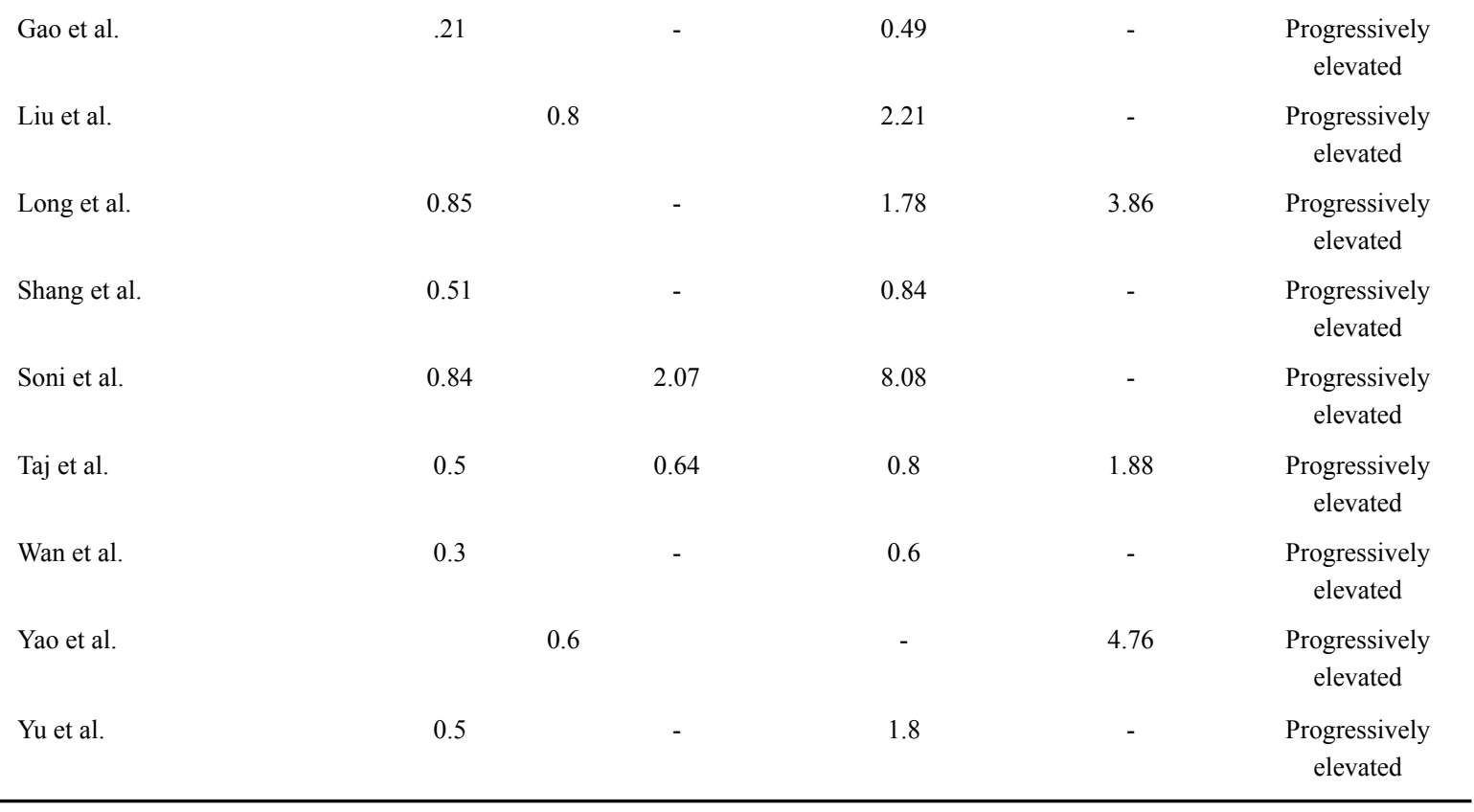

In total, 6,815 patients were screened to evaluate the relationship between D-dimer levels and the severity of COVID-19. 3,615 patients were identified to have mild to moderate conditions, 3,114 cases were severe, and 86 were critical. All 12 studies reported that D-dimer levels increased as the disease progressed. It was found that D-dimer values are higher in the critical category than in the severe category, and are likewise higher in the severe category than in the mild to moderate category. This shows that patient D-dimer levels are progressively elevated as the disease becomes more severe.

Table 5. Summary of the Mined D-dimer Values According to Disease Severity

\begin{tabular}{lccc}
\hline Disease Severity & No. of Patients & Median D-dimer $(\mu \mathrm{g} / \mathrm{L})$ & Normal range $(\leq 0.5 \mu \mathrm{g} / \mathrm{mL})$ comparison \\
\hline Mild to moderate & 3,615 & 0.505 & above normal \\
Severe & 3,114 & 0.8 & above normal \\
Critical & 86 & 3.86 & above normal \\
Total & $\mathbf{6 , 8 1 5}$ & $\mathbf{0 . 6 2}$ & above normal \\
\hline
\end{tabular}

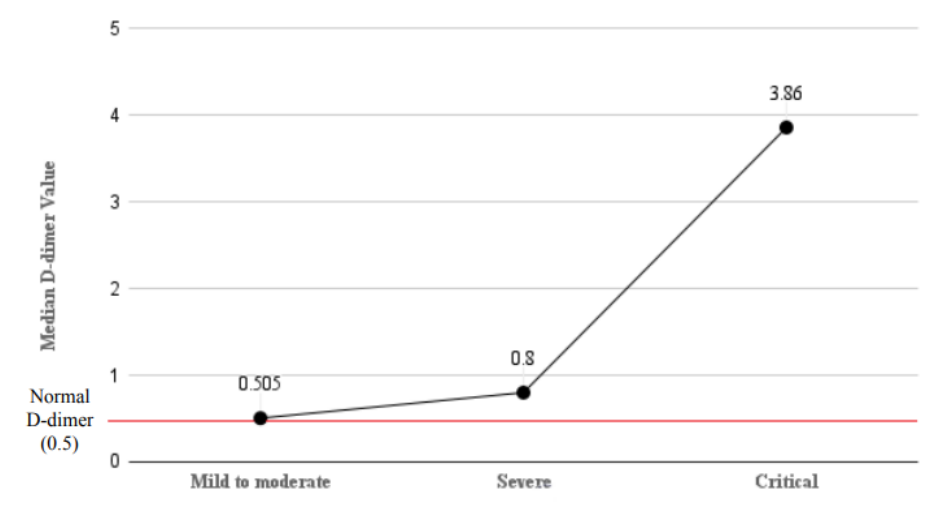

Fig. 2. Median D-dimer of Each Severity Classification

The median D-dimer for each severity classification was identified, and it was found that patients with mild to moderate conditions have a median D-dimer level of $0.505 \mu \mathrm{g} / \mathrm{mL}$, severe cases have a median D-dimer level of $0.8 \mu \mathrm{g} / \mathrm{mL}$, 
and critical cases exhibit the highest median D-dimer level with $3.86 \mu \mathrm{g} / \mathrm{mL}$. Compared to the normal D-dimer level $(\leq 0.5$ $\mu \mathrm{g} / \mathrm{mL}$ ), all three categories have median D-dimer levels that are above the normal range. The computed median D-dimer for all studies in all categories is $0.62 \mu \mathrm{g} / \mathrm{mL}$, which is also above normal.

Table 6. No. of Studies Within and Above the Normal Range According to Disease Severity

\begin{tabular}{lcc}
\hline Disease Severity & No. of Patients & Median D-dimer $(\mu \mathrm{g} / \mathrm{mL})$ \\
\cline { 2 - 3 } & Normal median D-dimer $(\leq 0.5 \mu \mathrm{g} / \mathrm{mL})$ & Elevated median D-dimer $(>0.5 \mu \mathrm{g} / \mathrm{mL})$ \\
\hline Mild to moderate & 6 & 6 \\
Severe & 4 & 7 \\
Critical & 0 & 3 \\
\hline
\end{tabular}

In the mild to moderate category, 6 studies presented D-dimer values that were above the normal range $(\leq 0.5$ $\mu \mathrm{g} / \mathrm{mL}$ ), while 6 studies identified normal D-dimer values. In the severe category, 7 studies were out of the normal range due to increased D-dimer levels, and only 4 studies determined normal values. In the critical category, 3 out of 3 studies showed D-dimer values that were above normal, and no study was within the normal range.

\subsubsection{Relationship of D-dimer levels and the mortality of COVID-19}

Of the 20 studies reviewed in this paper, 11 studies analyzed the relationship between D-dimer levels and the mortality of COVID-19. These studies documented the D-dimer values of COVID-19 patients who survived and died from the disease. The relative findings are presented in Table 8 and Table 9.

Table 7. Distribution of Patients based on Disease Mortality

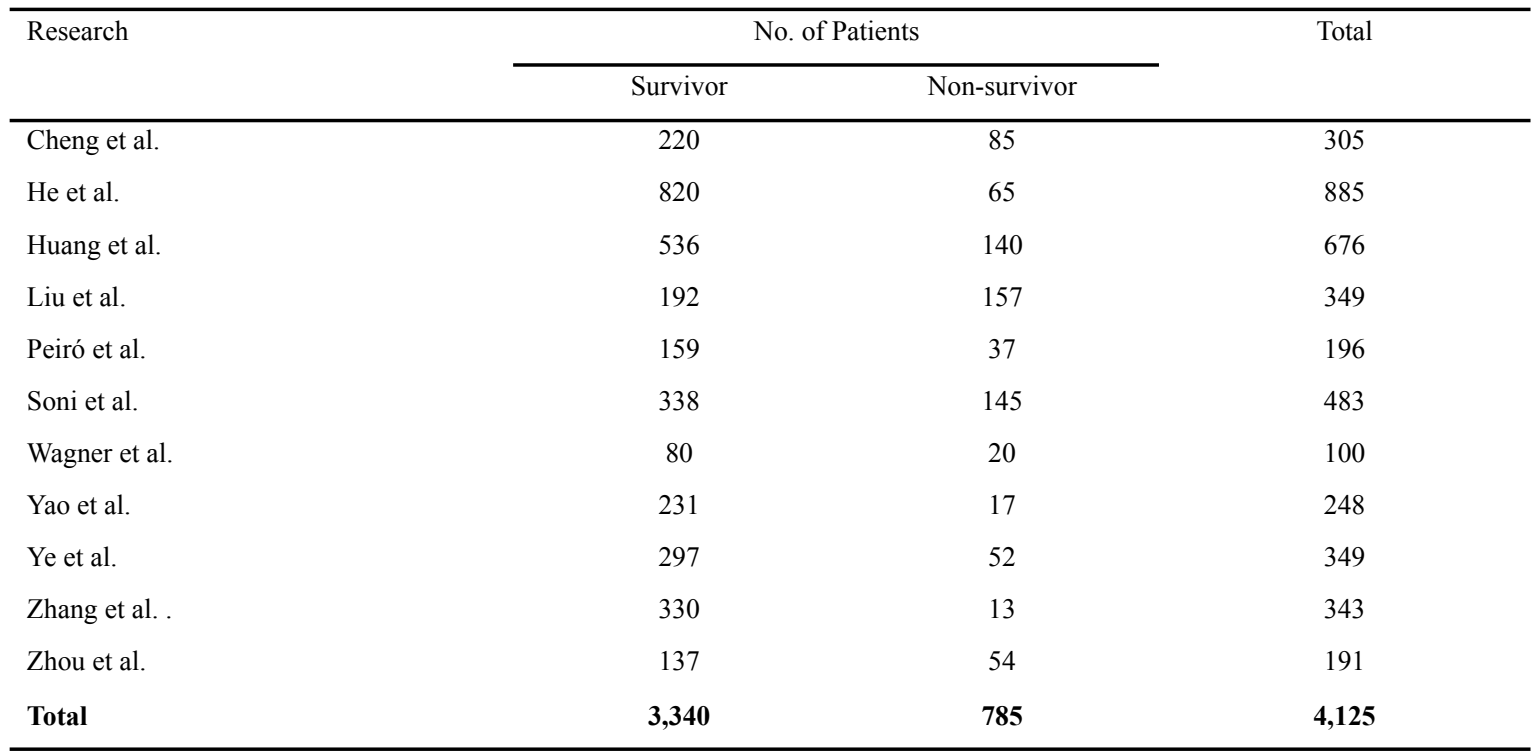

Table 8. The Mined D-dimer Values According to Survival

\begin{tabular}{llr}
\hline Research & Median D-dimer $(\mu \mathrm{g} / \mathrm{mL})$ & D-dimer level \\
\hline
\end{tabular}




\begin{tabular}{lccl}
\hline & Survivor & Non-survivor & \\
\hline Cheng et al. & 0.60 & 5.3 & higher in non-survivor \\
He et al. & 0.80 & 3.92 & higher in non-survivor \\
Huang et al. & 0.95 & 10.33 & higher in non-survivor \\
Liu et al. & 0.95 & 17.83 & higher in non-survivor \\
Peiró et al. & 0.618 & 1.834 & higher in non-survivor \\
Soni et al. & 0.94 & 6.34 & higher in non-survivor \\
Wagner et al. & 3.13 & 12.30 & higher in non-survivor \\
Yao et al. & 1.02 & 6.21 & higher in non-survivor \\
Ye et al. & 0.40 & 29.44 & higher in non-survivor \\
Zhang et al. & 0.41 & 4.76 & higher in non-survivor \\
Zhou et al. & 1.0 & 42.20 & higher in non-survivor \\
\hline
\end{tabular}

In total, 4,125 patients were screened to evaluate the relationship between D-dimer levels and the mortality of COVID-19, in which 3,340 patients survived and 785 patients died. All 11 studies reported that D-dimer levels of COVID-19 patients are higher in non-survivors. D-dimer values were found to be lower in patients who survived. No study documented higher D-dimer for survivors as compared to the non-survivor population.

Table 9. Summary of the Mined D-dimer Values According to Survival

\begin{tabular}{lccc}
\hline Patient Classification & No. of Patients & Median D-dimer $(\mu \mathrm{g} / \mathrm{mL})$ & Normal range $(\leq 0.5 \mu \mathrm{g} / \mathrm{mL})$ comparison \\
\hline Survivor & 3,340 & 0.94 & above normal \\
Non-survivor & 785 & 6.34 & above normal \\
Total & $\mathbf{4 , 1 2 5}$ & $\mathbf{2 . 4 8 2}$ & above normal \\
\hline
\end{tabular}

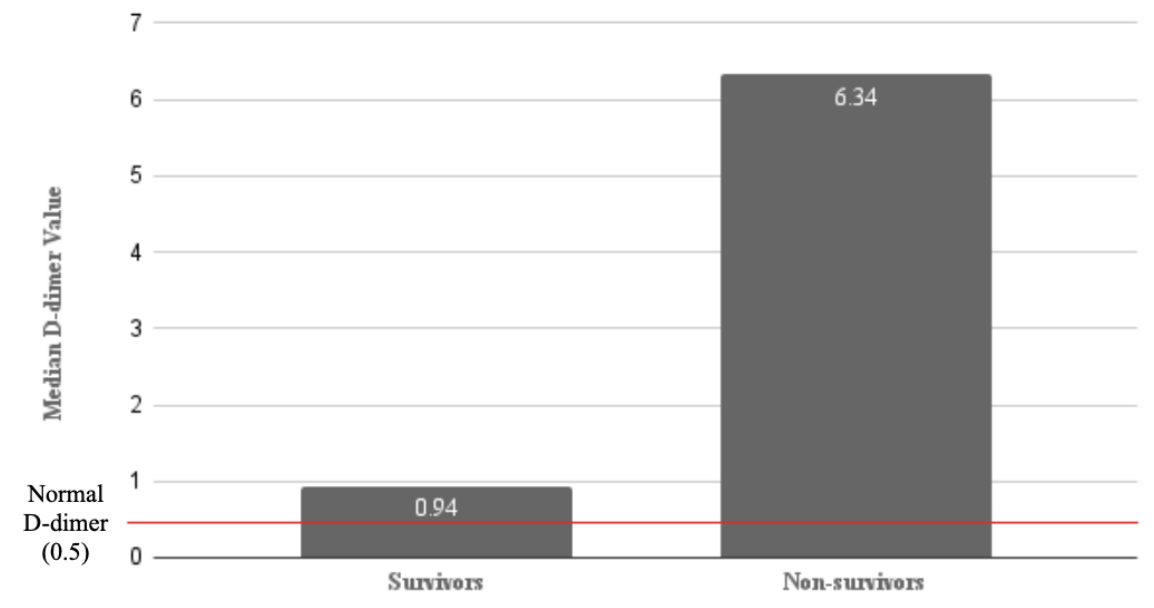

Fig. 3. Median D-dimer of Each Survival Group

The computed median D-dimer levels of the survivor and non-survivor groups are $0.94 \mu \mathrm{g} / \mathrm{mL}$ and $6.34 \mu \mathrm{g} / \mathrm{mL}$, respectively. These median D-dimer values show that $\mathrm{D}$-dimer levels are higher in cases of death than in cases of survival. Compared to the normal D-dimer level $(\leq 0.5 \mu \mathrm{g} / \mathrm{mL})$, both the survivor and non-survivor groups presented median D-dimer 
levels that are above the normal range. The computed median D-dimer for all studies in both categories is $2.482 \mu \mathrm{g} / \mathrm{mL}$, which is above normal.

Table 10. No. of Studies Within and Above the Normal Range According to Survival

\begin{tabular}{lcc}
\hline Patient classification & No. of Studies & Median D-dimer $(\mu \mathrm{g} / \mathrm{L})$ \\
\cline { 2 - 3 } & Normal median D-dimer $(\leq 0.5 \mu \mathrm{g} / \mathrm{mL})$ & Elevated median D-dimer $(>0.5 \mu \mathrm{g} / \mathrm{mL})$ \\
\hline Survivor & 3 & 8 \\
Non-survivor & 0 & 11 \\
\hline
\end{tabular}

In the survivor group, 8 studies presented D-dimer values that were above the normal range $(\leq 0.5 \mu \mathrm{g} / \mathrm{mL})$, and only 3 studies identified normal D-dimer values. In the non-survivor category, all 11 studies were elevated, and no study was within the normal range.

\subsection{Diseases associated with elevated D-dimer in COVID-19 patients}

Several studies found that certain diseases are associated with COVID-19 patients whose D-dimer values were above normal. In the study by Berger et al. (2020), complications such as hypertension, hyperlipidemia, coronary artery disease, and chronic kidney disease are frequent among COVID-19 patients with elevated D-dimer. Zhang et al. (2020) reported that patients with $\mathrm{D}$-dimer levels $\geq 2.0 \mu \mathrm{g} / \mathrm{mL}$ had a higher incidence of underlying diseases such as diabetes, hypertension, coronary heart disease, and stroke.Liu et al. and Soni et al. discussed the association of diabetes and increased D-dimer in COVID-19 patients. According to Liu et al. (2020), death was 11 times higher as a risk in patients with diabetes than those without diabetes. In addition, the study by Soni et al. (2020) established the significant difference between diabetic and non-diabetic patients with a p-value of $<0.001$, obtaining median D-dimer values of $1.68 \mu \mathrm{g} / \mathrm{mL}$ and 0.8 $\mu \mathrm{g} / \mathrm{mL}$, respectively. According to He et al. (2021), D-dimer was associated with disseminated intravascular coagulation, where patients who experienced DIC had D-dimer levels with a median of $16.14 \mu \mathrm{g} / \mathrm{mL}$, and patients who have not experienced DIC had a median of 0.83 (p-value $<0.001$ ). In the study by Yu et al. (2020), patients who showed elevated levels of D-dimer were associated with a higher mortality rate of community-acquired pneumonia.

\subsection{Reasons for increased D-dimer values in COVID-19 patients}

Several studies elaborated on the possible reasons why D-dimer values are increased in patients with COVID-19. Some of these studies specified why D-dimer levels are higher in more severe conditions of the disease, as well as in cases of mortality. According to Berger et al. (2020), pathological reports show that massive amounts of thrombi or blood clots formed within the vascular system are observed in COVID-19 patients, thereby resulting in increased D-dimer levels. It was also stated that hospitalized COVID-19 patients categorized with severe infection are found to have high incidence of thromboembolic events. Both the studies by Gao et al. (2020) and Yu et al. (2020) reported that COVID-19 patients with severe conditions exhibit elevated D-dimer due to abnormal coagulation. In the study by Liu et al. (2020), it was discussed that coagulation can be activated as a physiological response to infection. Therefore, activation of coagulation due to the high affinity of SARS-CoV-2 with vascular endothelial cells contributes to elevated D-dimer levels. Wan et al. (2020) noted that D-dimer concentrations are more elevated in patients with severe COVID-19 than those with mild conditions due to the presence of a hypercoagulable state and secondary fibrinolysis in vivo. Similarly, Ye et al. (2020) indicated that the manifestation of blood hypercoagulable state in COVID-19 patients with pneumonia results in increased D-dimer levels. Critically ill patients exhibit higher D-dimer due to frequent clotting disorders and micro thrombotic formation in the peripheral blood vessels. As discussed by Long et al. (2020), increased D-dimer in more severe cases of COVID-19 could be attributed to the dynamic coagulation or hypercoagulation that patients may experience. D-dimer levels gradually increase when a patient is already in a hyper fibrinolytic state since D-dimer is the degradation product of fibrinolysis. Fu et al. (2020) posited that a systemic inflammatory response will occur in COVID-19 patients, resulting in cytokine release, 
problems with blood components, and coagulation factor activation, which may lead to increased D-dimer. Dong et al. (2020) observed higher D-dimer levels in patients with severe cases of the disease than in non-severe cases, indicating the presence of sustained inflammatory response and defective coagulatory mechanisms upon infection with SARS-CoV-2. According to Zhou et al. (2020), 90\% of the COVID-19 patients diagnosed with pneumonia had an increased coagulation activity, in which D-dimer levels were elevated. He et al. (2020) identified three possible reasons for increased D-dimer values for COVID-19 patients. First, infection may result in the release of proinflammatory cytokines, thereby causing an inflammatory storm. Second, COVID-19 patients have different degrees of hypoxia, and inflammation could cause thrombosis or increased oxygen consumption. Lastly, severe infection, or acute inflammation caused by sepsis, may affect blood coagulation, thereby activating the coagulation cascade and promoting thrombosis. All of these are causes of increased D-dimer.

\subsection{Discussion}

A total of 20 studies were systematically reviewed to elaborate on the association of D-dimer levels with the disease severity and mortality of COVID-19, and D-dimer levels were found to be predominantly elevated in patients with COVID-19. The majority of the studies presented median D-dimer values that were above the normal concentration $(\leq 0.5$ $\mu \mathrm{g} / \mathrm{mL})$.

Twelve studies were analyzed for the relationship between D-dimer levels and the severity of COVID-19, and all of them reported that D-dimer values become more elevated in higher degrees of the disease (i.e. severe and critical cases than in mild to moderate cases). 3,615 patients were identified to have mild to moderate conditions, 3,114 cases were severe, and 86 were critical. The normal D-dimer level of $\leq 0.5 \mu \mathrm{g} / \mathrm{mL}$ was compared with the respective median D-dimer levels of the three severity categories. It was found that all computed median D-dimer values are above the normal value, as patients with mild to moderate conditions have a median D-dimer level of $0.505 \mu \mathrm{g} / \mathrm{mL}$, severe cases with $0.8 \mu \mathrm{g} / \mathrm{mL}$, and critical cases with the highest median D-dimer level of $3.86 \mu \mathrm{g} / \mathrm{mL}$. Specifically, 6 out of 12 studies in the mild to moderate category, 7 out of 12 studies in the severe category, and 3 out of 3 studies in the critical category, presented median D-dimer levels that were above normal.

For the association of D-dimer levels with COVID-19 mortality, a total of 11 studies were reviewed. It was found that the non-survivors $(n=785)$ have higher levels of D-dimer as compared to the survivors $(n=3340)$, with computed median values of $6.34 \mu \mathrm{g} / \mathrm{mL}$ and $0.94 \mu \mathrm{g} / \mathrm{mL}$, respectively. These median D-dimer values show that both the survivor and non-survivor groups are above the normal value. In the non-survivor population, all studies presented median D-dimer levels that were above normal. On the other hand, 8 out of 11 studies showed elevated median D-dimer levels in the survivor population, while 3 were within the normal range.

Six studies established that patients with elevated D-dimer levels had a higher COVID-19 incidence in the presence of an underlying disease. The following conditions increase the risk of acquiring COVID-19: hypertension, hyperlipidemia, coronary heart disease, chronic kidney disease, stroke, disseminated intravascular coagulation, community-acquired pneumonia, and diabetes.

\section{Conclusion}

D-dimer measurement is a laboratory test that may be beneficial in evaluating COVID-19 patients. Although all COVID-19 patients exhibit high D-dimer values disregarding the severity and mortality, elevated D-dimers are predominantly observed in severe to critical disease states and in cases of death. In addition, patients with pre-existing conditions such as hypertension, hyperlipidemia, coronary heart disease, stroke, disseminated intravascular coagulation, community-acquired pneumonia, and diabetes may also have elevated D- dimers. Thus, despite the fact that D-dimer is a nonspecific biomarker for COVID-19, it can be critical for disease identification and surveillance. 


\section{Recommendations}

Further investigation is recommended on other important laboratory biomarkers to determine their association with COVID-19, namely C-reactive protein, lactate dehydrogenase, and cardiac troponin. Different risk variables must also be accurately analyzed via long-term observations for better prognosis and outcome. Additional research is needed to corroborate the findings in different geographic regions and to determine the correlation between elevated D-dimer concentrations and COVID-19 severity and mortality, as well as its association with disease progression and prognosis. It is highly suggested to have a larger sample size for future studies since a small sample size provides limited interpretations which could affect the statistical outcomes of the study. A meta-analysis is also advised for future systematic reviews to establish the limitations in this study and to improve the credibility of the results. Moreover, medical centers should collaborate and form a control group and create follow-ups for patients who have been released or cured to ensure reliability of results, rigorous monitoring of prospective biomarkers, and prevention of clinical deterioration..

\section{Acknowledgements}

First and foremost, we would like to thank our Heavenly Father for providing us with wisdom, guidance, determination, and good health through the completion of this research. We would like to extend our deepest gratitude to our thesis adviser, Mr. Gamaliel Issamar De Vera, for constantly giving us inputs and advice that have helped us finish this paper. We sincerely appreciate the supervision and knowledge that he imparted upon us. We would also like to thank the University of Santo Tomas for giving us the necessary virtues that have helped us diligently progress in this research, and the esteemed faculty members of the Department of Medical Technology for supervising us and imparting their knowledge through the years. Finally, to our family and friends who have always given their support and prayers, we are truly grateful to have wonderful people surrounding us with understanding, care, and love. Their continuous encouragement to keep moving forward has become an important driving force in the completion of our thesis. We dedicate this study to the Field of Medical Technology.

\section{References}

Berger, J. S., Kunichoff, D., Adhikari, S., Ahuja, T., Amoroso, N., Aphinyanaphongs, Y., Cao, M., Goldenberg, R., Hindenburg, A., Horowitz, J., Parnia, S., Petrilli,C., Reynolds, H., Simon, E., Slater, J., Yaghi, S., Yuriditsky, E., Hochman, J., Horwitz, L. I., 2020. Prevalence and outcomes of D-dimer elevation in hospitalized patients with COVID-19. Arteriosclerosis, Thrombosis, and Vascular Biology, 40, p. $2539-2547$.

Bounds, E., Kok, S. J., 2020. D dimer, in “StatPearls”. StatPearls Publishing, Florida.

Cheng, A., Hu, L., Wang, Y., Huang, L., Zhao, L., Zhang, C., Liu, X., Xu, R., Liu, F., Li, J., Ye, D., Wang, T., Lv, Y., Liu, Q., 2020. Diagnostic performance of initial blood urea nitrogen combined with D-dimer levels for predicting in-hospital mortality in COVID-19 patients. International Journal of Antimicrobial Agents, 56.

Connors, J.M., Levy, J.H. 2020. COVID-19 and its implications for thrombosis and anticoagulation. The American Society of Hematology, 135, p. 2033-2040.

D-dimer test. MedlinePlus. https://medlineplus.gov/lab-tests/d-dimertest/\#: : text=A\%20D\%2Ddimer\%20test $\% 20$ is,other\%20 parts $\% 20$ of $\% 20$ the $\% 20$ body.

Mucha, S. R., Dugar, S., McCrae, K., Joseph, D., Bartholomew, J., Sacha, G. L., Militello, M., 2020. Coagulopathy in COVID-19: Manifestations and management. Cleveland Clinic Journal of Medicine, 87, p. 461-468.

Fu, J., Kong, J., Wang, W., Wu, M., Yao, L., Wang, Z., Jin, J., Wu, D., Yu, X., 2020. The clinical implication of dynamic neutrophil to lymphocyte ratio and D-dimer in COVID-19: A retrospective study in Suzhou China. Thrombosis Research, 192, p. 3-8.

Gao, Y., Li, T., Han, M., Li, X., Wu, D., Xu, Y., Zhu, Y., Liu, Y., Wang,. X., Wang, L., 2020. Diagnostic utility of clinical laboratory data determinations for patients with the severe COVID-19. Journal of Medical Virology, 92, pp. 791- 796.

He, X., Yao, F., Chen, J., Wang, Y., Fang, X., Lin, X., Long, H., Wang, Q., Wu, Q., 2021. The poor prognosis and influencing factors of high D-dimer levels for COVID-19 patients. Scientific Reports, 11, p. 1-7.

Huang, Y., Lyu, X., Li, D., Wang, L., Wang, Y., Zou, W., Wei, Y., Wu, X., 2020. A cohort study of 676 patients indicates D-dimer is a critical risk factor for the mortality of COVID-19. PLOS ONE, 15, p. 1-11.

Liu, J., Zhang, S., Wu, Z., Shang, Y., Dong, X., Li, G., Zhang, L., Chen, Y., Ye, X., Du, H., Liu, Y., Wang, T., Huang, S., Chen, L., Wen, Z., Qu, J., Chen, D., 2020. Clinical outcomes of COVID-19 in Wuhan, China: A large cohort study. Annals of Intensive Care, 10.

Long, H., Nie, L., Xiang, X., Li, H., Zhang, X., Fu, X., Ren, H., Liu, W., Wang, Q., Wu, Q., 2020. D-dimer and prothrombin time are the significant indicators of severe COVID-19 and poor prognosis. BioMed Research International, 2020, p. 1-10. 
Peiró, Ó., Carrasquer, A., Sánchez-Gimenez, R., Lal-Trehan, N., Del-Moral-Ronda, V., Bonet, Gil, Fort-Gallifa, I., Picó-Plana, E., Bastón-Paz, N., Gutiérrez, C., Bardaji, A. 2020. Biomarkers and short-term prognosis in COVID-19. Biomarkers, 26, p. 119-126.

Rothan, H. A., Byrareddy, S. N. 2020. The epidemiology and pathogenesis of coronavirus disease (COVID-19) outbreak. Journal of Autoimmunity, 109.

Shang, W., Dong, J., Ren, Y., Tian, M., Li, W., Hu, J., Li, Y. 2020. The value of clinical parameters in predicting the severity of COVID-19. Journal of Medical Virology, 92, p. 2188-2192.

Soni, M. Gopalakrishnan, R., Vaishya, R., Prabu, P. 2020. D-dimer level is a useful predictor for mortality in patients with COVID-19: Analysis of 483 cases. Diabetes \& Metabolic Syndrome: Clinical Research \& Reviews, 14, p. 2245-2249.

Taj, S., Ahmed, Q. 2021. Role of hematological parameters in the stratification of COVID- 19 disease severity. Annals of Medicine and Surgery, 62, p. 68-72.

Wagner, J., Garcia-Rodriguez, V., Yu, A., Dutra, B., DuPont, A., Cash, B., Farooq, A., 2020. Elevated D-dimer is associated with multiple clinical outcomes in hospitalized Covid-19 patients: A retrospective cohort study. SN Comprehensive Clinical Medicine, 2, p. $2561-2567$.

Wan, S., Xiang, Y., Fang, W., Zheng, Y., Li, B., Hu, Y., Lang, C., Huang, D., Sun, Q., Xiong, Y., Huang, X., Lv, J, Luo, Y., Shen, L., Yang, H., Huang, G., Yang, R. 2020. Clinical features and treatment of COVID-19 patients in northeast Chongqing. Journal of Medical Virology, 92, p. 797-806.

World Health Organization, 2020. Coronavirus. https://www.who.int/health- topics/coronavirus\#tab=tab_1.

World Health Organization, 2021. WHO coronavirus (COVID-19) Dashboard. https://covid19.who.int/

Yao, Y., Cao, J., Wang, Q., Shi, Q., Kai, L., Luo, Z., Chen, X., Chen, S., Yu, K., Huang, Z., Hu, B. 2020. D-dimer as a biomarker for disease severity and mortality in COVID-19 patients: A case control study. Journal of Intensive Care, 8.

Ye, W., Chen, G., Li, X., Lan, X., Ji, C., Hou, M., Zhang, D., Zeng, G., Wang, Y., Xu, C., Lu, W., Cui, R., Cai, Y., Huang, H., Yang, L. 2020. Dynamic changes of D-dimer and neutrophil-lymphocyte count ratio as prognostic biomarkers in COVID-19. Respiratory Research, 21, p.1-7.

Yu, H., Qin, C., Chen, M., Wang, W., Tian, D. 2020. D-dimer level is associated with the severity of COVID-19. Thrombosis Research, 195 , p. $219-225$.

Zhang, J., Dong, X., Cao, Y., Yuan, Y., Yang, Y., Yan, Y., Akdis, C. A., Gao, Y., 2020. Clinical characteristics of 140 patients infected with SARS- CoV-2 in Wuhan, China. European Journal of Allergy and Clinical Immunology, 75, p. 1730-1741.

Zhang, L., Yan, X., Fan, Q, Liu, H., Liu, X., Liu, Z., 2020. D-dimer levels on admission to predict in-hospital mortality in patients with Covid-19. Journal of Thrombosis and Haemostasis, 18, p. 1324-1329.

Zhou, F., Yu, T., Du, R., Fan, G., Liu, Y., Liu, Z., Xiang, J., Wang, Y., Song, R., Gu, X., Guan, L., Wei, Y., Li, H., Wu, X., Xu, J., Tu, S., Zhang, Y., Chen, H., Chao, B., 2020. Clinical course and risk factors for mortality of adult inpatients with COVID-19 in Wuhan, China: A retrospective cohort study. The Lancet, 395, p. 1054-1062.

Zu, Y., Jiang, M., Xu, P., Chen, W., Ni, Q., Lu, G., Zhang, L., 2020. Coronavirus disease 2019 (COVID-19): A perspective from China. Radiology, 296.

\section{Appendix A. PRISMA 2020 Checklist}

PRISMA 2020 Checklist

\begin{tabular}{|c|c|c|c|}
\hline $\begin{array}{l}\text { Section and } \\
\text { Topic } \\
\end{array}$ & Item & Checklist item & $\begin{array}{l}\text { Location } \\
\text { where item } \\
\text { is reported }\end{array}$ \\
\hline \multicolumn{4}{|c|}{ | } \\
\hline Title & 1 & Identify the report as a systematic review. & $i$ \\
\hline \multicolumn{4}{|l|}{ ABSTRACT } \\
\hline Abstract & 2 & See the PRISMA 2020 for Abstracts checklist. & ii \\
\hline \multicolumn{4}{|l|}{ INTRODUCTION } \\
\hline Rationale & 3 & Describe the rationale for the review in the context of existing knowledge. & N/A \\
\hline Objectives & 4 & Provide an explich statement of the objective(s) or question(s) the review addresses. & p.3 \\
\hline \multicolumn{4}{|l|}{ METHODS } \\
\hline Eligibility criteria & 5 & Specily the inclusion and exclusion criteria for the review and how studies were grouped for the syntheses. & p. 39 \\
\hline $\begin{array}{l}\text { Information } \\
\text { sources }\end{array}$ & ${ }^{6}$ & $\begin{array}{l}\text { Specify all databases, registers, websites, organisations, reference lists and other sources searched or consulted to identify studies. Specily the } \\
\text { date when each source was last searched or consulted. }\end{array}$ & p.38 \\
\hline Search strategy & 7 & Present the full search strategies for all databases, registers and websites, including any fiters and limits used. & p.38 \\
\hline Selection process & 8 & $\begin{array}{l}\text { Specify the methods used to decide whether a study met the inclusion criteria of the review, including how many reviewers screened each record } \\
\text { and each report retrieved, whether they worked independently, and if appicable, details of automation tools used in the process. }\end{array}$ & p. 40 \\
\hline $\begin{array}{l}\text { Data collection } \\
\text { process }\end{array}$ & ${ }^{9}$ & $\begin{array}{l}\text { Specify the methods used to collect data from reports, including how many reviewers collected data from each report, whether they worked } \\
\text { independently, any processes for obtaining or confirming data from study investigators, and if applicable, details of automation tools used in the } \\
\text { process. }\end{array}$ & p. 40 \\
\hline \multirow[t]{2}{*}{ Data items } & 10a & $\begin{array}{l}\text { List and define all outcomes for which data were sought. Specify whether all results that were compatitble with each outcome domain in each } \\
\text { study were sought (e.g. for all measures, time points, analyses), and if not, the methods used to decide which results to collect. }\end{array}$ & p. 40 \\
\hline & $10 \mathrm{~b}$ & $\begin{array}{l}\text { List and define all other variables for which data were sought (e.g. participant and intervention characteristics, funding sources). Describe any } \\
\text { assumptions made about any missing of unclear information. }\end{array}$ & N/A \\
\hline $\begin{array}{l}\text { Study risk of bias } \\
\text { assessment }\end{array}$ & 11 & $\begin{array}{l}\text { Specify the methods used to assess risk of bias in the included studies, including details of the tool(s) used, how many reviewers assessed each } \\
\text { study and whether they worked independently, and if applicable, details of automation tools used in the process. }\end{array}$ & N/A \\
\hline Effect measures & 12 & Specily for each outcome the effect measure(s) (e.g. risk ratio, mean difference) used in the synthesis or presentation of results. & N/A \\
\hline \multirow[t]{6}{*}{$\begin{array}{l}\text { Synthesis } \\
\text { methods }\end{array}$} & 13a & $\begin{array}{l}\text { Describe the processes used to decide which studies were eligible for each synthesis (e. } . \text { tabulating the study intervention characteristics and } \\
\text { comparing against the planned groups for each synthesis (nem } \# 5) \text { ). }\end{array}$ & p.42 \\
\hline & 136 & $\begin{array}{l}\text { Describe any methods required to prepare the data for presentation or synthesis, such as handling of missing summary statistics, or data } \\
\text { conversions. }\end{array}$ & N/A \\
\hline & 13c & Describe any methods used to tabulate or visually display results of individual studies and syntheses. & N/A \\
\hline & ${ }^{130}$ & $\begin{array}{l}\text { Describe any methods used to synthesize results and provide a rationale for the choice(s). If meta-analysis was performed, describe the } \\
\text { model(s), method(s) to identifit the presence and extent of statistical heterogeneity, and sofware package(s) used. }\end{array}$ & N/A \\
\hline & $13 \mathrm{e}$ & Describe any methods used to explore possible causes of heterogeneity among study results (e.g. subgroup analysis, meta-regression). & N/A \\
\hline & $13 \mathrm{f}$ & Describe any sensitivity analyses conducted to assess robustness of the synthesized results. & N/A \\
\hline $\begin{array}{l}\text { Reporting bias } \\
\text { assessment }\end{array}$ & 14 & Describe any methods used to assess risk of bias due to missing results in a synthesis (arising from reporting biases). & N/A \\
\hline $\begin{array}{l}\text { Certainty } \\
\text { assessment }\end{array}$ & 15 & Describe any methods used to assess certainty (or confidence) in the body of evidence for an outcome. & N/A \\
\hline
\end{tabular}


PRISMA 2020 Checklist

\begin{tabular}{|c|c|c|c|}
\hline $\begin{array}{l}\text { Section and } \\
\text { Topic }\end{array}$ & them & Checklist item & $\begin{array}{l}\text { Location } \\
\text { where item } \\
\text { is reported }\end{array}$ \\
\hline \multicolumn{4}{|l|}{ RESULTS } \\
\hline \multirow[t]{2}{*}{ Study selection } & 16a & $\begin{array}{l}\begin{array}{l}\text { Describe the results of the search and selection process, from the number of records identfied in the search to the number of studies included in } \\
\text { the review, ideally using a flow diagram. }\end{array}\end{array}$ & p.42 \\
\hline & $16 \mathrm{~b}$ & Cite studies that might appear to meet the inclusion criteria, but which were excluded, and explain why they were excluded. & N/A \\
\hline $\begin{array}{l}\text { Study } \\
\text { characteristics }\end{array}$ & 17 & Cite each included study and present its characteristics. & p.44 \\
\hline $\begin{array}{l}\text { Risk of bias in } \\
\text { studies }\end{array}$ & 18 & Present assessments of risk of bias for each included study. & N/A \\
\hline $\begin{array}{l}\text { Resuls of } \\
\text { individual studies }\end{array}$ & 19 & $\begin{array}{l}\text { For all outcomes, present, for each study: (a) summary statistics for each group (where appropriate) and (b) an effect estimate and its precision } \\
\text { (e.g. confidencee/credible interval), ideally using structured tables or plots. }\end{array}$ & p.44 \\
\hline \multirow{4}{*}{$\begin{array}{l}\text { Results of } \\
\text { syntheses }\end{array}$} & $20 \mathrm{a}$ & For each synthesis, briefly summarise the characteristics and risk of bias among contributing studies. & N/A \\
\hline & $20 \mathrm{~b}$ & $\begin{array}{l}\begin{array}{l}\text { Present results of all statistical syntheses conducted. II meta-analysis was done, present for each the summary estimate and its precision (e.g. } \\
\text { conffidence/credible interval) and measures of statistical heterogenenety. If comparing groups, describe the direction of the effect. }\end{array} \\
\end{array}$ & N/A \\
\hline & $20 \mathrm{c}$ & \begin{tabular}{|l|l|} 
Present resulls of all investigations of possible causes of heterogeneity among study resulls. \\
\end{tabular} & N/A \\
\hline & 200 & \begin{tabular}{|l} 
Present results of all sensitivity analyses conducted to assess the robustness of the synthesized results. \\
\end{tabular} & N/A \\
\hline Reporting biases & 21 & Present assessments of risk of bias due to missing results (arising from reporting biases) for each synthesis assessed. & N/A \\
\hline $\begin{array}{l}\text { Certainty of } \\
\text { evidence }\end{array}$ & 22 & Present assessments of certainty (or confidence) in the body of evidence for each outcome assessed. & N/A \\
\hline \multicolumn{4}{|l|}{ DISCUSSION } \\
\hline \multirow[t]{4}{*}{ Discussion } & $23 \mathrm{a}$ & \begin{tabular}{|l|l} 
Provide a general interpretation of the results in the contex of other evidence. \\
\end{tabular} & p. 100 \\
\hline & $23 \mathrm{~b}$ & Discuss any limitations of the evidence included in the review. & N/A \\
\hline & $23 \mathrm{c}$ & Discuss any limitations of the review processes used. & N/A \\
\hline & 23d & Discuss implications of the results for practice, policy, and future research. & p. 102 \\
\hline \multicolumn{4}{|c|}{ OTHER INFORMATION } \\
\hline \multirow{3}{*}{$\begin{array}{l}\text { Registration and } \\
\text { protocol }\end{array}$} & $24 \mathrm{a}$ & Provide registration information for the review, including register name and registration number, or state that the review was not registered. & N/A \\
\hline & $24 \mathrm{~b}$ & Indicate where the review protocol can be accessed, or state that a protocol was not prepared. & N/A \\
\hline & 240 & Describe and explain any amendments to information provided at registration or in the protocol. & N/A \\
\hline Support & 25 & Describe sources of financial or non-financial support for the review, and the role of the funders or sponsors in the review. & N/A \\
\hline $\begin{array}{l}\text { Competing } \\
\text { interests }\end{array}$ & 26 & \begin{tabular}{|l|l|} 
Declare any competing interests of review authors. \\
\end{tabular} & N/A \\
\hline $\begin{array}{l}\text { Availability of } \\
\text { data, code and } \\
\text { other materials }\end{array}$ & 27 & $\begin{array}{l}\text { Report which of the following are publicly available and where they can be found: template data collection forms; data extracted from included } \\
\text { studies; data used for all analyses; analytic code; any other materials used in the review. }\end{array}$ & N/A \\
\hline
\end{tabular}

